\title{
Concept and operation of Schottky emitter without suppressor electrode
}

\author{
A. K. Dokaniaa) and P. Kruit \\ Faculty of Applied Science, Delft University of Technology, Lorentzweg 1, 2628 CJ Delft, The Netherlands
}

(Received 20 May 2009; accepted 12 October 2009; published 18 November 2009)

\begin{abstract}
The Schottky electron emitter is the most frequently used electron source in electron microscopes. A suppressor electrode around the emitter is usually employed to prevent emission from the shank of the cathode. A concept of operating the Schottky emitter without the suppressor electrode is proposed with the aim of lowering the potential of the extractor electrode. Simulation results show that if the suppressor electrode is removed, then the same field as for the standard configuration can be obtained at the tip apex at an extraction voltage of $2265 \mathrm{~V}$ instead of $5000 \mathrm{~V}$. The total emission from the shank region is calculated by estimating the emission area of the shank, taking into the account the different work functions of the crystal facets. The total emission for typical operating parameters is calculated to rise from 500 to $668 \mu \mathrm{A}$. The total emission from the shank and the filament of the Schottky emitter is measured experimentally in two different configurations, which match with the simulated results. The measured total emission of 450-750 $\mu \mathrm{A}$ confirms the idea that a Schottky emitter can be operated without suppressor, all the more so because the power at the extractor aperture is even reduced as a result of the lower acceleration voltage. () 2009 American Vacuum Society. [DOI: 10.1116/1.3258657]
\end{abstract}

\section{INTRODUCTION}

The Schottky emitter is known for its high brightness and current stability. ${ }^{1,2}$ In a Schottky emitter, the useful emission comes from the $\mathrm{W}(100)$ facet at the very end of the tip. The emission from all the other parts of the tip (conical and cylindrical) called shank emission is suppressed by a suppressor electrode, which is usually an integral part of a Schottky emitter unit. The disadvantage of the suppressor electrode is that the negative potential at the suppressor electrode reduces the field at the apex of the emitter tip considerably. The potential of the extractor electrode has to be increased to compensate this reduced field. The typical potential applied to the extractor and suppressor electrodes are $5 \mathrm{kV}$ and -300 $\mathrm{V}$, respectively, for a tip-extractor distance of $500 \mu \mathrm{m}$. There have been efforts to operate the Schottky emitter at lower extraction voltages for its application in an array of microcolumns. ${ }^{3,4}$ To operate the Schottky emitter at low extraction voltage, the field at the tip should not change along with other parameters such as temperature and work function. The field at the tip has to be maintained to get the same angular current density and brightness. This can be done by bringing the extractor plate closer to the tip. In Ref. 5 Kim et al. discussed the operation of Schottky emitters below $1 \mathrm{kV}$ of extractor voltage, which were operated at a tip-extractor distance of 50-100 $\mu \mathrm{m}$ with 1-2 $\mu \mathrm{m}$ thick silicon extractors with an aperture of $5 \mu \mathrm{m}$. However, such small tipextractor distance and aperture size require very stringent tip positional stability, for a slight tip movement can give rise to a considerable change in the total electron emission. Moreover, a Si extractor of 1-2 $\mu \mathrm{m}$ thickness in close proximity to the tip at $1800 \mathrm{~K}$ is susceptible to thermal effects.

To lower the potential of the extractor electrode it is proposed to operate the Schottky emitter without the suppressor

\footnotetext{
${ }^{a)}$ Electronic mail: a.k.dokania@tudelft.nl
}

electrode. This concept would have several advantages over the conventional Schottky emitter. There would be no issue of alignment of the tip with respect to the suppressor electrode. It would also make the whole Schottky emitter unit less voluminous. The lower extraction potential would result in lower heat dissipation on the extractor electrode, thus less susceptible to thermal drifts and outgassing. The suppressorless Schottky emitter allows also for a higher tip radius emitter which has better emission and shape stability without increasing the extraction voltage beyond $5 \mathrm{kV}$. The above advantages would make it easier to construct a Schottky emitter array for multibeam lithography.

A suppressor electrode will have no influence on the electron optics as long as the field at the tip remains the same. It is shown in Ref. 6 that the angular intensity distribution is similar for different suppressor voltages if the field at the tip is kept constant by adjusting the anode voltage. Therefore, the Schottky emitter can be operated at much lower extraction voltage if there is no suppressor electrode at all. For the suppressorless Schottky emitter configuration, it is important to know the total emission from the conical and cylindrical shank and the filament, which would be falling on the extractor in the absence of the suppressor electrode. The total emission current in the suppressorless configuration has first been simulated and then measured in two different extractor configurations: with and without an aperture to distinguish between shank and facet current from the tip. The total emission from a typical Schottky emitter varies from $\sim 40$ to $500 \mu \mathrm{A}$ depending upon the operating conditions. The emission from the facet varies from 4 to $40 \mu \mathrm{A}$.

\section{SIMULATION METHOD}

CPO software ${ }^{7}$ is commonly used for the modeling of electrostatic, magnetostatic, and ray tracing problems. The CPO program uses a boundary element method or charge density 




FIG. 1. Standard Schottky emitter configuration, used for the simulation.

method to approximate the partial derivatives for the potential and is suitable for problem with large scale difference in geometries of various electrodes. The various geometries of the electrodes i.e., emitter, suppressor electrode and extractor electrode are defined in the program. The geometry has a two-dimensional cylindrical symmetry around the emitter axis, shown in Fig. 1. In this configuration, the tip-extractor distance is $508 \mu \mathrm{m}$, the bore diameter of extractor and suppressor electrodes are $380 \mu \mathrm{m}$, and the tip-suppressor distance is $242 \mu \mathrm{m}$. The tip apex is approximated to a truncated hemisphere of radius $500 \mathrm{~nm}$. The radius of this hemisphere is considered the tip radius $(r)$ and the truncated part (usually $0.3 r$ ) is called facet of the tip. The emitter apex was positioned far away from the boundary of electrodes as the potential at the domain boundary may affect the overall solution. In the beginning of the simulation, the emitter is grounded and the extractor and suppressor are biased at $5 \mathrm{kV}$ and $-300 \mathrm{~V}$, respectively. Subsequently the bias voltage at the extractor and suppressor are changed to estimate different electric fields at the apex of the tip.

The total shank emission has been calculated using the standard Schottky emission theory. With this theory the local current density on the surface can be calculated if the local temperature, field, and work function (WF) are known. The field along the conical and cylindrical shank [Fig. 2(a)] is determined locally at the interval of $100 \mathrm{~nm}$ by the simulation program. While calculating the current density from the conical shank, the work function of different crystallographic orientations, i.e., the four $\mathrm{W}(100) / \mathrm{ZrO}$ lobes $[\mathrm{WF}=2.95 \mathrm{eV}$ (Ref. 8)] and polycrystalline tungsten [WF=4.6 eV (Ref. 9)] have been taken into consideration. For the cylindrical shank, the work function of polycrystalline tungsten is considered. Then using the Schottky equation ${ }^{8}$ for each $100 \mathrm{~nm}$ wide ring, current density is calculated. The total emission has been estimated using the total surface area of the emitter excluding the $\mathrm{ZrO}_{2}$ lump. The diameter and length of the cylindrical shank are assumed to be $125 \mu \mathrm{m}$ and $1.12 \mathrm{~mm}$,
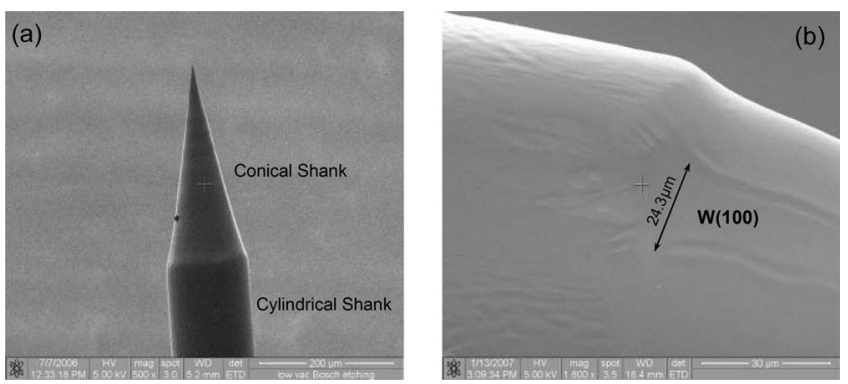

FIG. 2. Standard Schottky emitter. (a) Total shank region, (b) the band of $\mathrm{W}(100)$ on the conical shank.

respectively. The length of the conical shank is estimated to be $265 \mu \mathrm{m}$. The area of the four W(100) lobes on the conical shank has been estimated with the help of a scanning electron microscopy picture [Fig. 2(b)].

\section{SIMULATION RESULTS}

The tip shape and the geometry used for the above simulation method were verified by comparing the field enhancement factor $\beta$, given by Eq. (1) (Ref. 8) to the field enhancement factor obtained by simulation for the standard configuration with a suppressor,

$$
\beta=\left[\frac{\mathrm{LSA}-\left(1-V_{\text {sup }} / V_{\text {ext }}\right)(\text { LTA-0.0068) }}{0.366 \mathrm{LSA}(r)^{0.758}}\right] \frac{1}{\mathrm{~cm}},
$$

where LTA is the distance between the tip and the extractor, LSA is the distance between the suppressor and the extractor electrode, and $r$ is the tip radius (all units in centimeters). The electric field at the apex of the tip is studied for the standard Schottky tip shape and geometry (i.e., LSA $=750 \mu \mathrm{m}$, LTA $=508 \mu \mathrm{m}, r=0.5 \mu \mathrm{m}, V_{\text {ext }}=5 \mathrm{kV}$, and $\left.V_{\text {sup }}=-300 \mathrm{~V}\right)$. The field values for the standard configuration, the suppressorless configuration from the simulation and the theoretical field value using Eq. (1) are plotted in Fig. 3. The $\beta$ obtained from Eq. (1) and from the simulated

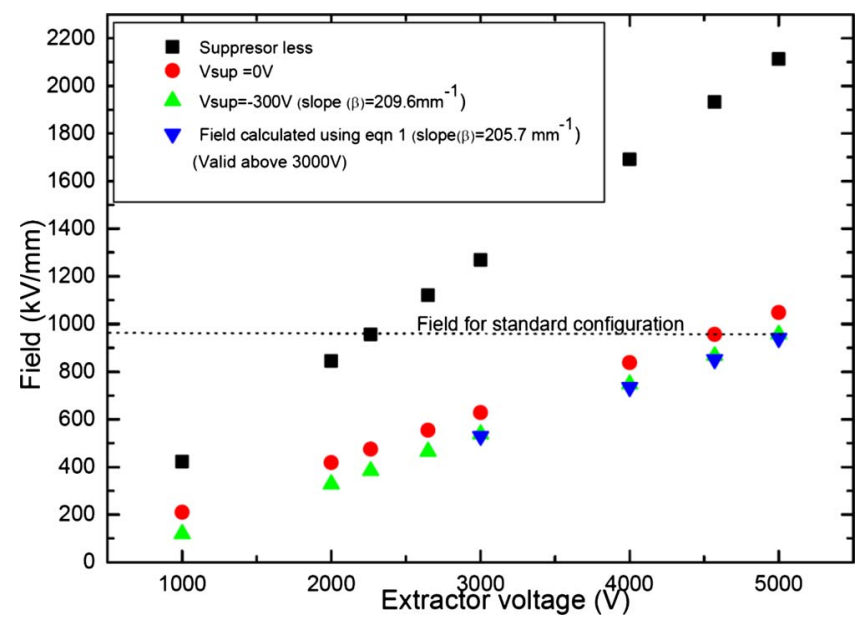

FIG. 3. (Color online) Field value obtained by CPO simulation and from Eq. (1) for various extractor voltages. 



FIG. 4. Simulated field lines at $V_{\text {ext }}=5 \mathrm{kV}$ and (a) $V_{\text {sup }}$ $=-300 \mathrm{~V}$. (b) No suppressor.

results matches within the accuracy of $\pm 1.9 \%$, thus validating our simulation.

For the standard configuration, the field at the center of the apex can be deduced from Fig. 3 to be $0.96 \mathrm{~V} / \mathrm{nm}$. The same field as that of a standard configuration can be achieved by lowering the extraction voltage to $2265 \mathrm{~V}$ in the suppressorless configuration. The field at the apex without suppressor at the extraction voltage of $5 \mathrm{kV}$ is approximately 2.1 $\mathrm{V} / \mathrm{nm}$. With zero suppressor voltage, the field of $0.96 \mathrm{~V} / \mathrm{nm}$ can be obtained at the extractor potential of $4570 \mathrm{~V}$. The difference between zero suppressor voltage and no suppressor is considerable.

Figure 4 shows the equipotential lines around the apex of the tip in the standard and suppressorless configuration. From the figures, it is evident that the line density increases as the suppressor electrode is removed. Since the tip is far from the boundary, the boundary effect in the simulation can be neglected.

The total shank emission for various configurations has been calculated and shown in Table I. The conical shank emission increases by more than $30 \%$ for the suppressorless configuration at $2265 \mathrm{~V}$ extractor voltage compared to the standard configuration as the increased field led to increased Schottky effect. However the total Joule heating (emission $\times V_{\text {ext }}$ ) of the extractor electrode would be less than that of the standard configuration as the potential is reduced by more than $50 \%$. The total cylindrical emission assuming there were no $\mathrm{W}(100)$ planes is estimated to be only a few microamperes and therefore the increase is not significant in comparison to the emission from the conical part. However, if there were $\mathrm{W}(100)$ planes then the emission would be 4.22 $\mathrm{mA}$ for $2265 \mathrm{~V}$ of extractor voltage, as shown within the

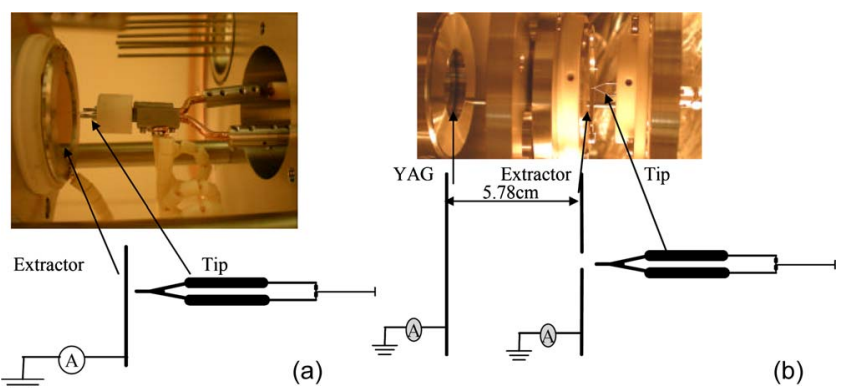

FIG. 5. (Color online) (a) Schematic of the experimental setup with the Schottky emitter and extractor electrode to measure the total emission (a) without an aperture with tip-extractor distance of $850 \mu \mathrm{m}$ (b) with an aperture with tip-extractor distance of $600 \mu \mathrm{m}$

bracket in Table I. It has to be noted that in case of a standard configuration in the presence of a suppressor electrode, no emission from the cylindrical shank takes place. Sections IV and $\mathrm{V}$ discuss the total emission and shank current measurements.

\section{EXPERIMENTAL METHODS}

The setup consists of a Schottky emitter without its suppressor electrode and aligned to a molybdenum extractor plate in front of it. The tip-extractor distances of 600 and $850 \mu \mathrm{m}$ were maintained for two different configurations, shown in Fig. 5. In the first configuration the total emission current and current on the extractor plate is measured. In the second configuration, an extractor with an aperture of $380 \mu \mathrm{m}$ was placed to distinguish between facet and shank current. The tip-extractor distance is set in such a way that the facet current will pass through the aperture. The total current falling on the extractor electrode is measured by a multimeter attached to the extractor electrode outside the chamber. In this setup the extractor was externally grounded, and the emitter is connected to the field emission gun supply unit (FGSU). The FGSU was connected to the computer and used to control the heating current for the emitter and to measure the total emission from the tip. The reference negative potential for the emitter was controlled by connecting it to a Heinzinger high voltage supply. To minimize outgassing, the system was baked to $250{ }^{\circ} \mathrm{C}$ and equipped with a TKS mass spectrometer to check the gases released from $m / e$ ranging from 1 to 50 during outgassing.

TABLE I. Shank current estimation for various configurations.

\begin{tabular}{lcc}
\hline \hline Configuration $\left(V_{\text {ext }}, V_{\text {sup }}\right)$ & $\begin{array}{c}\text { Conical shank current } \\
(\mathrm{A})\end{array}$ & $\begin{array}{c}\text { Cylindrical shank current } \\
\text { (A) [with W(100) plane] }\end{array}$ \\
\hline Standard, 5000-300 V & $4.98 \times 10^{-4}$ & $\ldots$ \\
Standard, 5000, 0 V & $5.57 \times 10^{-4}$ & $2.48 \times 10^{-7}\left(2.62 \times 10^{-3}\right)$ \\
Suppressorless, 5000 V & $11.3 \times 10^{-4}$ & $5.26 \times 10^{-7}\left(5.58 \times 10^{-3}\right)$ \\
Suppressorless, 2265 V & $6.68 \times 10^{-4}$ & $3.98 \times 10^{-7}\left(4.22 \times 10^{-3}\right)$ \\
Suppressorless with coating of Mo, 2265 V & $1.03 \times 10^{-7}$ & $3.98 \times 10^{-7}$ \\
\hline
\end{tabular}




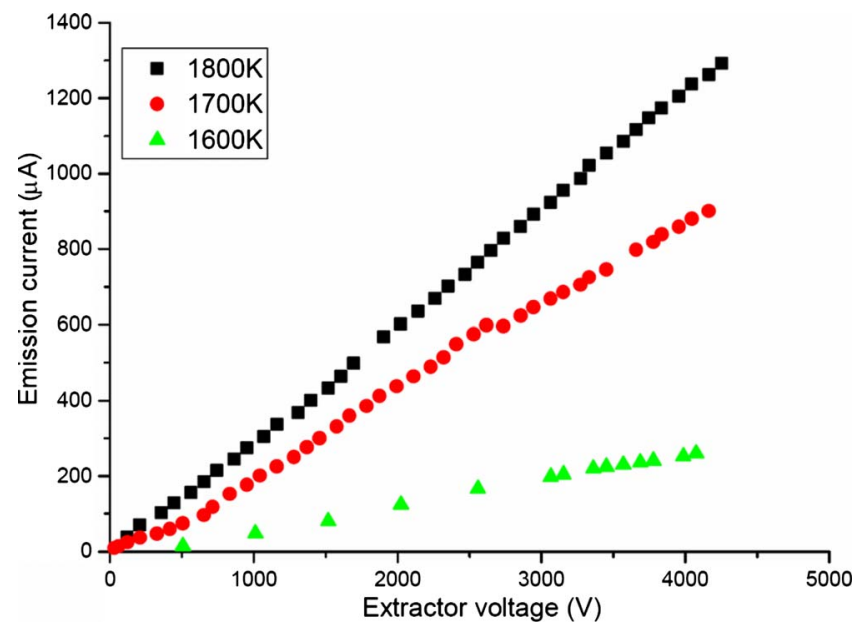

FIG. 6. (Color online) Total emission current without suppressor electrode with respect to varying extraction voltage at various operating temperatures.

\section{EXPERIMENTAL RESULTS}

First the total emission from the heating filament of a broken Schottky emitter (without emitter) is measured. With increasing extraction voltage, the emission current increases and then saturates to $14 \mu \mathrm{A}$ at an extraction voltage of 300 $\mathrm{V}$. The thermionic emission from the filament has been estimated using the temperature distribution across the filament ${ }^{10}$ and the measured experimental value matches well. Therefore from the simulation results of total emission in Table I, it can be corroborated that the major part of total emission in case of suppressorless configuration would be from the cone and the shank of the monocrystalline tip.

The total emission from a Schottky emitter without suppressor electrode has been measured using the setup explained in Fig. 5(a). Figure 6 shows the two total emission measurements as a function of the extraction voltage at different temperatures. From the measurements shown in Fig. 6, it can be seen that the total emission at $\sim 2300 \mathrm{~V}$ (the potential at which the field at the tip is the same as in a standard configuration) is $\sim 700 \mu \mathrm{A}$, which matches the simulation result of $668 \mu \mathrm{A}$ at $2265 \mathrm{~V}$, discussed in Sec. IV reasonably. This result also rules out the possibility of having fully developed W(100) sides in the cylindrical shank, else a total emission of more than $4 \mathrm{~mA}$ could be expected. The deviation in the simulation and measured result could be due to the over-/underestimation of the total emitting area, i.e., the four W(100) lobes on the cone are longer than estimated; the difference in the configuration of tip-extractor distance and finally there may be some $\mathrm{W}(100)$ facets on the surface of cylindrical part. ${ }^{11}$

The linear relationship between the total emission and the extraction voltage $(V)$ is somewhat surprising. Pure space charge limited thermionic emission would be expected to rise with $V^{3 / 2}$ following the Child-Langmuir equation. Pure Schottky emission would rise even faster with extraction voltage. However, in other situations such a linear rise has also been observed. ${ }^{12,13}$



FIG. 7. (Color online) Spectrum of gases released due to different emission currents and energies.

It would be desirable to separate the total current in terms of facet current and shank current and measure angular current density or even brightness of the beam from the facet. The second experiment was done using an extractor with an aperture, shown in Fig. 5(b). The distance between tipextractor is set in such a way that all the facet current will pass through the aperture and gets collected at the YAG (yttrium aluminum garnet) screen. One of the issues encountered in this experiment was the electron stimulated desorption (ESD) of gases, possibly from the extractor, Macor, or the chamber walls. Since the pressure rose above the safe operating pressure of Schottky emitters, the extractor voltage could not be increased beyond $750 \mathrm{~V}$. Figure 7 shows the spectrum from a well baked system and results of electron induced desorption. After identifying the gases, without emission, the same experiment was done with the emission and increasing extraction voltage to see the outgassing pattern of the gases. The main gases are $\mathrm{H}_{2}, \mathrm{CO}, \mathrm{CO} 2$, and $\mathrm{CH}_{4} / \mathrm{CH}_{3}$. Similar desorbed gases were also reported for other systems such as stainless steel, aluminum, tungsten, molybdenum, etc. ${ }^{14-18}$ It has been noted that in the first configuration, ESD was not a problem. We conclude that in the second configuration the ESD can be attributed to the electrons hitting other parts of the setup than the extractor.

The problem of outgassing has been minimized by high temperature $\left(\sim 250{ }^{\circ} \mathrm{C}\right)$ baking of the system. The total emission and the extractor current measurements are shown in Fig. 8(a). The total emission measured was comparable to the previous measurement taking into account the increased tip-extractor distance and different tip radii. The facet current on the YAG screen could not be measured and no emission pattern was seen. This could be due to oxidation of the aluminum coating on the YAG screen during baking and/or the low energy of the electrons that could not penetrate through the dead layer. Since the tip-extractor distance is maintained in such a way that only the low angle emission from the facet $\left(<6^{\circ}\right)$ passes through the aperture of the extractor, the facet current can be approximated by subtracting the extractor cur- 



Fig. 8. (Color online) (a) Total emission current without suppressor electrode with respect to varying extraction voltage at $1800 \mathrm{~K}$ with the configuration of Fig. 5(b), after hard bakeout. (b) Plot of $I_{\text {facet }}$ vs $V_{\text {ext }}$.

rent from the total emission to extractor current, shown in Fig. 8(b). With the estimate of the angle that passes the extractor, the measured facet current of $80 \mu \mathrm{A}$ may correspond to an angular intensity of $0.8-1 \mathrm{~mA} / \mathrm{sr}$, which is normal for a Schottky emitter.

\section{DISCUSSION}

Normally, the four W (100) lobes on the conical part develop during heating in the presence of an electric field, the mechanism of which can be found in Ref. 19. Coating the conical and cylindrical shanks with a material of higher work function than $\mathrm{W}(100) / \mathrm{ZrO}$ might prevent the formation of such high emission planes. The material to be coated should have melting point higher than the operating temperature, i.e., $1800 \mathrm{~K}$, comparable electronegativity for proper $\mathrm{ZrO}_{2}$ diffusion toward the apex, comparable thermal expansion, and lattice parameter as that of tungsten for better coating stability. After carefully analyzing a list of materials, the choice is narrowed down to molybdenum, which meets all the above requirements. The polycrystalline molybdenum has a work function of $4.6 \mathrm{eV} .{ }^{20}$ The prevention of formation of $\mathrm{Mo}(100)$ is imperative as $\mathrm{Mo}(100) / \mathrm{ZrO}$ has a work function of $2.1 \mathrm{eV},^{21}$ which is even lower than $\mathrm{W}(100) / \mathrm{ZrO}$. It is evident from the $\mathrm{W}-\mathrm{Mo}$ phase diagram ${ }^{22}$ that tungsten and molybdenum form a solid solution at all temperatures and concentrations without forming any intermetallic compounds. The details of the interdiffusion of molybdenum and tungsten are beyond the scope of this article and can be found in Refs. 23-25. The interdiffusion between Mo-W might prevent the crystallization of Mo in W(100) direction. $^{26,27}$ With the work function of $4.6 \mathrm{eV}$ for molybdenum, the total conical shank emission is calculated to be reduced by a factor of $10^{4}$ shown in Table I. Such W(100) plane can also be presented or developed on the cylindrical part, which needs to be checked experimentally.

One may argue that the energy spread at the lower voltage operation of the suppressorless Schottky emitter would increase due to the Boersch effect. In general, the emission process (intrinsic) and the Coulomb interactions of the emitted electrons (extrinsic) both contribute to the energy distribution of electrons. In Ref. 28, Bronsgeest et al. presented the way to distinguish the Boersch effect from the intrinsic energy distribution. Since the intrinsic energy distribution is a function of temperature, work function, and the field at the emitter, this distribution can be assumed to be the same in case of the suppressorless configuration. Most of the energy broadening due to the Boersch effect usually occurs in the crossover, in this case very close to the tip. A recent calculation by Bronsgeest et al. $^{29}$ shows that about $80 \%$ of the broadening occurs in the first $100 \mu \mathrm{m}$. This is where the energy of the electrons is mainly determined by the field on the facet, which is equal in the suppressorless configuration. Therefore it can be said that the energy broadening in the case of suppressorless Schottky emitter will not be significantly different from the standard Schottky emitter. The possible loss of brightness due to Coulomb interactions is a more complicated story, but easier to control, precisely because most of the effect occurs further away from the tip.

\section{CONCLUSION}

A Schottky emitter without the suppressor electrode could be operated at lower extraction voltage compared to the standard configuration. Simulation results show that if the suppressor electrode is removed, then the same field as for the standard configuration can be obtained at the tip apex at an extraction voltage of $2265 \mathrm{~V}$ instead of $5000 \mathrm{~V}$. The estimated total emission without the suppressor electrode rises to $668 \mu \mathrm{A}$ in comparison with $500 \mu \mathrm{A}$ in the standard configuration. However the total heating power dissipating on the extractor would be $40 \%$ lower than the standard configuration. The total emission without the suppressor electrode matches well with simulated results and rules out the presence of W(100) planes on the cylindrical shank. The increase in the total emission resulted in increased outgassing which was minimized but could not be eliminated by good baking of the vacuum system. The increased outgassing would not 
be an issue as the power dissipating on the extractor is still lower. However, if W(100) planes form after a long time on the cylindrical shank it would alter the emission results and would increase the power dissipating on the extractor. Such an increased emission could be avoided by coating the shank with suitable materials such as molybdenum.

\section{ACKNOWLEDGMENTS}

The authors gratefully acknowledge M. Bronsgeest and C. W. Hagen of TU Delft and G. A. Schwind of FEI for reading the manuscript and giving valuable suggestions.

${ }^{1}$ L. W. Swanson and N. A. Martin, J. Appl. Phys. 46, 2029 (1975).

${ }^{2}$ D. W. Tuggle and L. W. Swanson, J. Vac. Sci. Technol. B 3, 220 (1985).

${ }^{3}$ H. S. Kim, M. L. Yu, E. Kratschmer, B. W. Hussey, M. G. R. Thomson, and T. H. P. Chang, J. Vac. Sci. Technol. B 13, 2468 (1995).

${ }^{4}$ Y. W. Yau, R. F. W. Pease, A. A. Iranmanesh, and K. J. Polasko, J. Vac. Sci. Technol. 19, 1048 (1981).

${ }^{5}$ H. S. Kim, E. Kratschmer, M. L. Yu, M. G. R. Thomson, and T. H. P. Chang, J. Vac. Sci. Technol. B 12, 3413 (1994).

${ }^{6}$ David Tuggle, Ph.D. thesis, Oregon Graduate Center, 1984.

${ }^{7}$ See: http://www.electronoptics.com.

${ }^{8}$ L. W. Swanson and G. A. Schwind, in Handbook of Charged Particle Optics, edited by J. Orloff (CRC, Boca Raton, 1997).

${ }^{9}$ J. Richard A Chapman, Appl. Phys. (Berlin) 35, 2832 (1964).

${ }^{10}$ A. K. Dokania, J. F. M. Velthuis, Y. Zhang, and P. Kruit, J. Vac. Sci. Technol. B 25, 504 (2007).
${ }^{11}$ FEI data sheet for Schottky emitters.

${ }^{12}$ K. R. Williams and R.S. Muller, Tech. Dig. - Int. Electron Devices Meet. $1992,387$.

${ }^{13}$ R. T. Longo, Tech. Dig. - Int. Electron Devices Meet. 1980, 467.

${ }^{14}$ E. M. Williams, F. Le Normand, N. Hilleret, and G. Dominichini, Vacuum 35, 141 (1985).

${ }^{15}$ R. S. Vaughan Watkins and E. M. Williams, Vacuum 28, 459 (1978).

${ }^{16}$ D. Edwards, J. Vac. Sci. Technol. 15, 1586 (1978).

${ }^{17}$ G. E. Moore, J. Appl. Phys. 32, 1241 (1961).

${ }^{18}$ S. G. Kasabov, Vacuum 38, 1035 (1988).

${ }^{19}$ S. Fujita and H. Shimoyama, Phys. Rev. B 75, 235431 (2007).

${ }^{20}$ H. B. Michaelson, J. Appl. Phys. 48, 4729 (1977).

${ }^{21}$ H. Nakane, S. Satoh, and H. Adachi, J. Vac. Sci. Technol. B 23, 769 (2005).

${ }^{22}$ S. V. Nagender Naidu, A. M. Sriramamurthy, and P. R. Rao, in Binary Alloy Phase Diagram, edited by Thaddeus B. Massalski (Willem W Scott, Jr, ASM, Metals Park, 1990).

${ }^{23}$ G. Wahl and Jutz Demny, IEEE Conference Record of 1970 Thermionic Conversion Specialist Conference, 1970 (unpublished), p.95.

${ }^{24}$ R.Butz, W. Erley and H. Wagner, IEEE Conference Record of 1970 Thermionic Conversion Specialist Conference, 1970 (unpublished), p. 101.

${ }^{25}$ F. Roux, D. Ablitzer and A. Vignes, IEEE Conference Record of 1970 Thermionic Conversion Specialist Conference, 1970 (unpublished), p. 109.

${ }^{26}$ S. P. Murarka, Metallization-Theory and Practice for VLSI and ULSI (Butterworth- Heinemann, New York, 1993), p. 30.

${ }^{27}$ C. P. Flynn, J. Phys. F: Met. Phys. 18, L195 (1988).

${ }^{28}$ M. S. Bronsgeest, J. E. Barth, G. A. Schwind, L. W. Swanson, and P. Kruit, J. Vac. Sci. Technol. B 25, 2049 (2007).

${ }^{29}$ M. S. Bronsgeest and P. Kruit (unpublished). 